\title{
THE PERIOD CHANGES OF YY ERIDANI
}

\author{
C.-H. KIM AND J. H. JEONG \\ Dept. of Astron. \& Space Science, Chungbuk Nat'l. Uni., San 48, Gaeshindong, Cheongju \\ 361-763, Korea \\ kimch@astro.chungbuk.ac.kr,jeongjh@astro.chungbuk.ac.kr \\ O. DEMIRCAN AND Z. MUYESSEROULU \\ Ankara Uni. Obs., Dept. of Astron., Tandogan, Ankara, Turkey \\ demircan@dione.astro.science.ankara.edu.tr, muyes@dione.astro.science.ankara.edu.tr
}

AND

E. BUDDING

Central Insitute of Technology, P.O. Box 40740, Wellington, New Zealand budding@ee.cit.ac.nz

A total of eighteen times of minimum lights for YY Eri were determined from relatively new or unpublished photoelectric observations collected from Korea and Turkey. All minima available to us were intensively analyzed to deduce the character of period variation of YY Eri. It is either formed by a sinusoidal variation superimposed on an upward parabola, or a set of abrupt changes. The abrupt changes appeared to have alternatively occured in the pattern of two increases following one decrease, which may be an indication of sinusoidal variations rather than real sudden changes of period. Upward parabolic variation can be due to a secular period increase caused by mass transfer from less massive to more massive component. The sinusoidal character can arise from a third body or from a strong magnetic activity cycle. Long term sinusoidal light level variation in the light curves supports the cyclic magnetic activity effect on the orbital period. However, the third body hypothesis can not be ruled out by the present data. 